\title{
Electron-scale sheets of whistlers close to the magnetopause
}

\author{
G. Stenberg ${ }^{1}$, T. Oscarsson ${ }^{1}$, M. André ${ }^{2}$, A. Vaivads $^{2}$, M. Morooka ${ }^{2}$, N. Cornilleau-Wehrlin ${ }^{3}$, A. Fazakerley ${ }^{4}$, \\ B. Lavraud ${ }^{5}$, and P. M. E. Décréau ${ }^{6}$ \\ ${ }^{1}$ Department of Physics, Umeå University, Umeå, Sweden \\ ${ }^{2}$ Swedish Institute of Space Physics, Uppsala, Sweden \\ ${ }^{3}$ CETP/UVSQ, Vélizy, France \\ ${ }^{4}$ MSSL, University College, London, UK \\ ${ }^{5}$ CESR, Toulouse, France \\ ${ }^{6}$ LPCE et Université d'Orléans, France
}

Received: 18 January 2005 - Revised: 10 October 2005 - Accepted: 8 November 2005 - Published: 23 December 2005

\begin{abstract}
Whistler emissions close to the magnetopause on the magnetospheric side are investigated using the four Cluster spacecraft. The waves are found to be generated in thin (electron-scale) sheets moving with the plasma drift velocity. A feature in the electron data coincides with the waves; hot magnetospheric electrons disappear for a few satellite spins. This produces or enhances a temperature anisotropy, which is found to be responsible for the generation of the whistler mode waves. The high energy electrons are thought to be lost through the magnetopause and we suggest that the field lines, on which the waves are generated, are directly connected to a reconnection diffusion region at the magnetopause.
\end{abstract}

Keywords. Magnetospheric physics (Magnetopause, cusp and boundary layers) - Space plasma physics (Magnetic reconnection; Waves and instabilities)

\section{Introduction}

Recent discoveries of thin (electron scale) layers (André et al., 2004; Vaivads et al., 2004a) at the magnetopause have gained new insights into magnetopause physics and have increased the hope of using the multi-satellite mission Cluster (Escoubet et al., 2001) to solve the mystery of reconnection.

We present in this paper one more piece in the puzzle, showing that whistler mode waves observed close to the magnetopause on the magnetospheric side also occur in thin sheets. We are lucky enough to record generation regions on three out of four Cluster spacecraft and a detailed study reveals that the thin sheets, where wave generation is assumed to occur, pass the spacecraft with the plasma drift velocity. The whistler emissions also coincide with a conspicuous structure in the electron data; high energy magnetospheric electrons disappear for a few satellite spins (roughly $10 \mathrm{~s}$ ), which might indicate that the thin sheets are on field lines directly connected to the reconnection diffusion region. Waves

Correspondence to: G. Stenberg

(stenberg@space.umu.se) at much higher frequencies - about the electron plasma frequency - are also observed in association with the thin sheets. These waves are observed at the same time intervals as the whistler waves, which put serious constraints on the propagation and/or damping properties of both types of waves.

\section{Background and related observations}

The magnetopause is one of the most interesting regions in the magnetosphere: a thin boundary between the shocked magnetosheath plasma, originating from the solar wind, and the magnetospheric plasma. Through this boundary particles and energy from the solar wind are assumed to enter the magnetosphere through a process generally referred to as reconnection. The reconnection process allows fast conversion of magnetic field energy into kinetic energy of electrons and ions (Paschmann, 1979; Øieroset, 2001). Large efforts are put into the task of understanding the structure and dynamics of the magnetopause and to reveal the details of the energy transfer across the boundary. Improved computer simulations, together with multi-spacecraft observations, continuously provide new clues (e.g. Vaivads et al., 2004b; Scholer et al., 2003).

Different wave modes are suggested to play important roles in the reconnection process (Cattell et al., 1995; Rezeau and Belmont, 2001; Petkaki et al., 2003). In recent years, interest has been directed towards whistler mode waves. In this paper we will refer to these waves as whistlers, noting, however, that they are different from the lightning generated waves often referred to by the same name. Whistler mode waves are believed to provide the means for a reconnection rate high enough to agree with measurements (Deng and Matsumoto, 2001). Reports on whistler mode activity in the dayside solar wind-magnetospheric interaction region have been frequent since in-situ exploration of the magnetopause and the bow shock began. Starting in the 60's, a number of spacecraft have searched the area and electromagnetic waves in a broad frequency range have been commonly 
observed (e.g. Smith et al., 1967 (OGO-1); Olson et al., 1969 (OGO-3); Rodriguez and Gurnett, 1975 (Imp-6); Gurnett et al., 1979 (ISEE 1 and 2); LaBelle and Treumann, 1988 (AMPTE/IRM); Zhang et al., 1998a (Geotail); Pickett et al., 1999 (Polar); Maksimovic et al., 2001 (Cluster)). It is worth noting that the waves are present in large regions of space and are observed not only in direct association with the boundaries but also further away from them. However, there are reasons to believe that many of the observations still are linked to the magnetopause or bowshock, for example through the particle distributions responsible for the generation of the waves (e.g. Zhang et al., 1998a).

In this context observations of thin sheets of wave activity thousands of kilometers from the large magnetopause current sheet are an important piece of evidence. They confirm that electron scale physics is relevant not only at reconnection sites but also far away from them. If the waves could be directly related to processes at the boundary/reconnection site, we would also have established a way of monitoring micro-physics at the magnetopause by remote sensing.

\section{Data}

Data used in this study are recorded by the four Cluster spacecraft. The satellites have elliptical polar orbits with a perigee of 4 Earth radii $\left(R_{E}\right)$ and an apogee of $19.6 R_{E}$. The orbits are fixed with respect to the stars, that is, the apogee moves around the Earth as the Earth rotates around the Sun. The satellite orbits are arranged so that in some regions of large scientific interest, for example, the magnetopause, the satellites form an optimized tetrahedron. The spacecraft separation is changed during the mission and is about $100 \mathrm{~km}$ for the event analyzed below. The spacecraft are spin-stabilized with a spin period of $4 \mathrm{~s}$, and the instrumentation is identical on all four of them (Escoubet et al., 1997, 2001).

In this study we use six of the Cluster instruments. The wave analysis is based on time series data from the electric field experiment (EFW) (Gustafsson et al., 1997) and the search coil magnetometer (STAFF) (Cornilleau-Wehrlin et al., 1997, 2003). STAFF provides three orthogonal magnetic wave field components. EFW uses two pairs of spherical probes deployed on wire booms to measure two orthogonal electric field components in the spin plane. The probe-toprobe separation is $88 \mathrm{~m}$. Both instruments are run in burst mode on all four spacecraft during this event, which means a sampling rate of 450 samples/second. The signals are low pass filtered with a cutoff frequency of $180 \mathrm{~Hz}$. Furthermore, the STAFF Spectrum Analyser (STAFF-SA) gives electric and magnetic wave field spectral data in the frequency range $64 \mathrm{~Hz}-4 \mathrm{kHz}$.

The emissions at higher frequencies are captured by the WHISPER instrument. WHISPER (Décréau et al., 1997) can be run as a passive receiver but also has an active sounder that emits short pulses to stimulate the characteristic frequencies of the plasma. A receiver then detects the plasma echos in the frequency range $4-80 \mathrm{kHz}$, and provides a way of measuring the plasma density.

To investigate the electron distributions we use data from the electron experiment PEACE (Johnstone et al., 1997). PEACE consists of two sensors positioned at opposite sides of the spacecraft, providing a 3-D distribution for the full energy range every four seconds (one spin period). The ion data come from the CIS/HIA instrument (Rème et al., 1997). The CIS instrument is capable of delivering a 3-D distribution each spin period. CIS data are used to estimate the plasma drift velocity and to model the ion component of the plasma.

In addition we also use high resolution data (67.2 vectors/s) from the fluxgate magnetometer (FGM) (Balogh et al., 1997) to transform magnetic wave field data into a locally background magnetic field oriented coordinate system.

\section{A first look at the event}

At 03:31 UT on 2 March 2002, the Cluster spacecraft crossed the magnetopause at high latitude (GSE: $x=7.15 R_{E}$, $\left.y=3.31 R_{E}, z=8.39 R_{E}\right)$ and close to magnetic noon. Almost 2 min prior, the satellites encountered the wave emission playing the principal part in this paper. Figure 1 introduces the event as seen by Cluster 4 . The top panel shows the probe-to-spacecraft potential obtained from EFW, which is a measure of the plasma density (Pedersen et al., 2001). The outbound (magnetosphere to magnetosheath) magnetopause crossing is clearly seen as a sudden increase in density at 03:31 UT. In the second and third panels of Fig. 1 we present time-frequency spectrograms of the magnetic and electric wave fields during the same time period. The main subject of this study is the wave emission appearing in both these panels at about 03:29:30 UT. Figure 1 gives the impression that the waves of interest are observed close to the magnetopause, but provides no estimate of the distance. However, particle observations (Sect. 7) confirm that the waves are recorded in a boundary layer with a mixture of magnetospheric and magnetosheath plasma. Hence, the waves are detected sufficiently close to the magnetopause, in order for the environment to be affected by the magnetosheath plasma.

The electromagnetic nature of the waves is obvious from Fig. 1. The emission lasts about $20 \mathrm{~s}$ and the frequency ranges from $50 \mathrm{~Hz}$ to well above $200 \mathrm{~Hz}$, with a fairly sharp lower cutoff. Due to the sampling rate of 450 samples/s and the low-pass filtering at $180 \mathrm{~Hz}$, we do not capture the highest frequencies of the emission. However, in STAFF-SA data (not shown) the emission is seen up to about $400 \mathrm{~Hz}$. The background magnetic field strength, $\boldsymbol{B}_{\mathbf{0}}$, (from FGM) is about $30 \mathrm{nT}$ (GSE: $B_{0 x}=-22 \mathrm{nT}, B_{0 y}=7 \mathrm{nT}, B_{0 z}=17 \mathrm{nT}$ ), giving an electron gyrofrequency of $840 \mathrm{~Hz}$. The electron plasma frequency (from WHISPER) is $15-20 \mathrm{kHz}$, corresponding to a plasma density of $2.8-5.0 \mathrm{~cm}^{3}$. Assuming a proton-electron plasma the lower-hybrid frequency can be estimated to about $20 \mathrm{~Hz}$. Hence, the electromagnetic waves we record are within the whistler mode frequency range, 


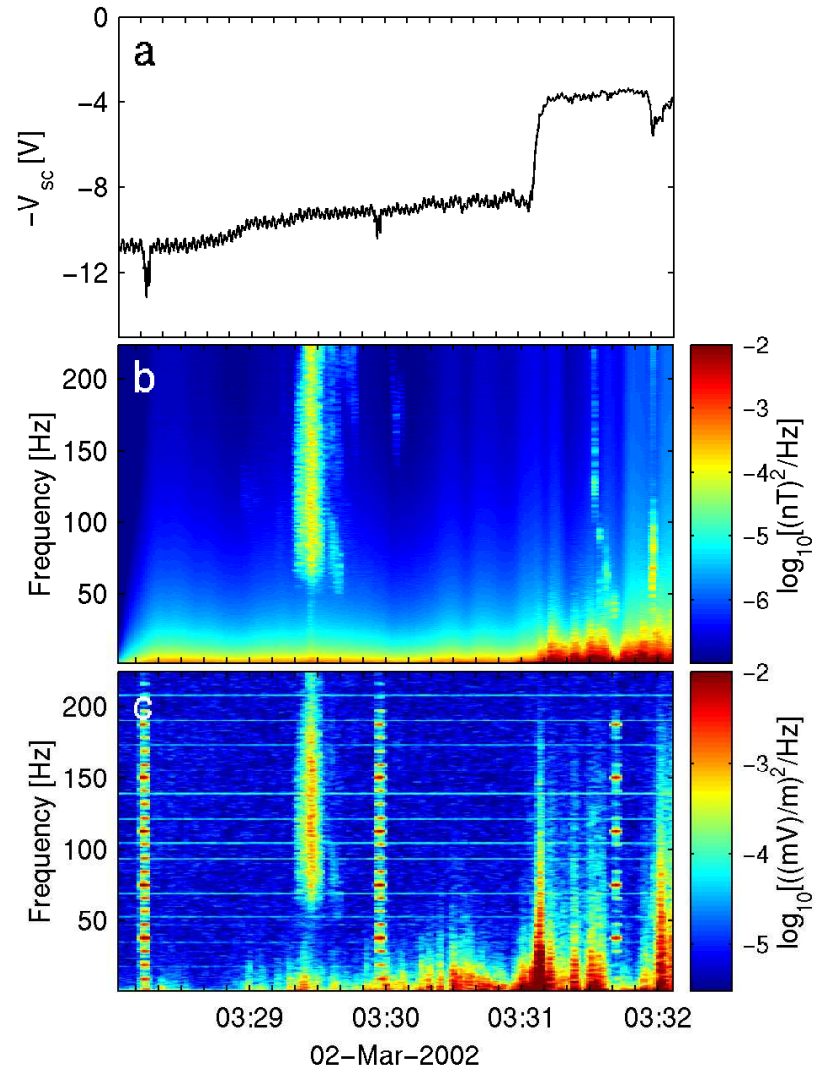

Fig. 1. (a) Probe-to-spacecraft potential (often referred to as the negative of the spacecraft potential, $\left(-V_{S C}\right)$ versus time. The plasma density is proportional to $-V_{S C}$ and the increase in density associated with the magnetopause crossing is clearly seen. The density on the magnetospheric side is about $3 \mathrm{~cm}^{-3}$, rising to about $20 \mathrm{~cm}^{-3}$ when the satellite enters the magnetosheath. Data are from EFW. (b) Total magnetic power spectral density (color coded) versus time and frequency (STAFF). The waves we concentrate on are seen at 03:29:30 UT. (c) Total electric power spectral density in the spin plane (color coded) versus time and frequency (EFW). The artificial-looking signals (e.g. at 03:30:00 UT) are caused by the active sounder WHISPER. All data are obtained from Cluster 4.

above the lower-hybrid frequency and below the electron gyrofrequency. At this point it could also be noted that in comparison with the rather monochromatic lion roars reported from this region of space (Baumjohann et al., 2000; Zhang et al., 1998b), the emission studied in this paper is broadbanded, lasts for a longer time and does not show any pronounced wave packet structure.

An investigation of the polarization further strengthens the mode identification. We transform the wave magnetic field into a local coordinate system with the $z$-axis along the background magnetic field $\boldsymbol{B}_{\mathbf{0}}$. The $y$-axis points roughly in the $y_{G S E}$ direction and the $x$-axis is in the $x_{G S E^{-}} z_{G S E^{-}}$ plane. Using $60 \mathrm{~s}$ of data we compute the cross spectral density of the two components perpendicular to $\boldsymbol{B}_{\mathbf{0}}$. We focus on the complex phase of the cross spectrum, $\eta_{x y}$, which is equivalent to the averaged phase difference between the

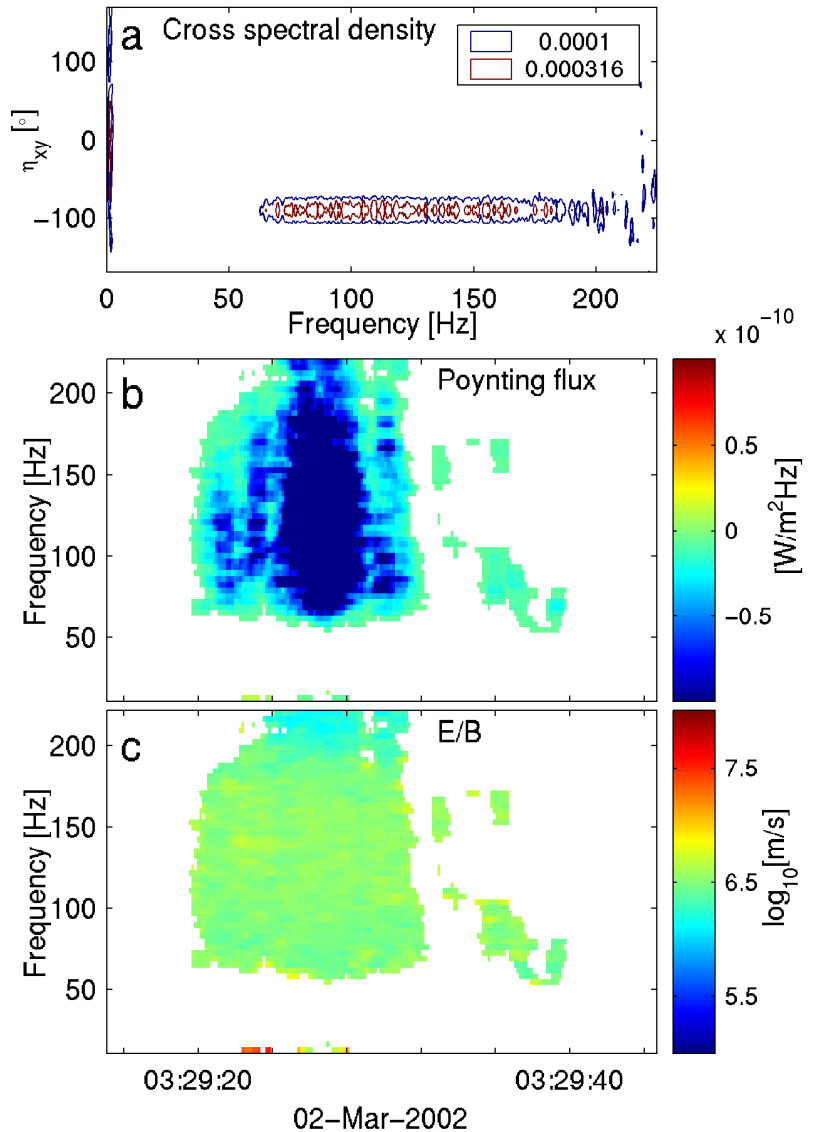

Fig. 2. (a) Contours showing the distribution of cross spectral density, $S_{x y}$ in frequency, $f$, and phase, $\eta_{x y}$. The cross spectrum is computed for the interval 03:29-03:30 UT using a record length of 1024 points and averaging over 101 time records. The cross spectral density is in units of $(\mathrm{nT})^{2} /{ }^{\circ} \mathrm{Hz}$. Integrating over all phase angles would yield the usual cross spectral density in $(\mathrm{nT})^{2} / \mathrm{Hz}$. (b) Parallel component of the Poynting flux, assuming $\boldsymbol{E} \times \boldsymbol{B}_{0}=0$. The whistler mode waves propagate anti-parallel to the background field. Timefrequency bins with a Poynting flux less than $2 \cdot 10^{-6} \mu \mathrm{W} / \mathrm{m}^{2} \mathrm{~Hz}$ are removed. (c) E/B versus time and frequency provides an estimation on the phase velocity, which for the waves of interest is about $5 \cdot 10 \mathrm{~m} / \mathrm{s}$. STAFF and EFW data are used.

two perpendicular magnetic wave field components. The cross spectral density is plotted versus phase and frequency in the top panel of Fig. 2. In the frequency range of interest, $\eta_{x y} \approx-90^{\circ}$, which corresponds to the right-handed polarization we expect for whistler waves. The coherence is very close to one in the frequency range $60-180 \mathrm{~Hz}$.

From a Poynting flux calculation we conclude that the whistler mode waves propagate anti-parallel to the ambient field. Since the observations are made in the Northern Hemisphere this correspond to waves propagating away from the Earth and towards the magnetopause. The parallel component of the Poynting flux, $P_{\|}$, is presented in the second panel of Fig. 2. $\left|P_{\|}\right|$is found, assuming that the wave electric field $(\boldsymbol{E})$ is perpendicular to $\boldsymbol{B}_{\mathbf{0}}$, which is a reasonable approximation for nearly anti-parallel propagating whistlers. The 


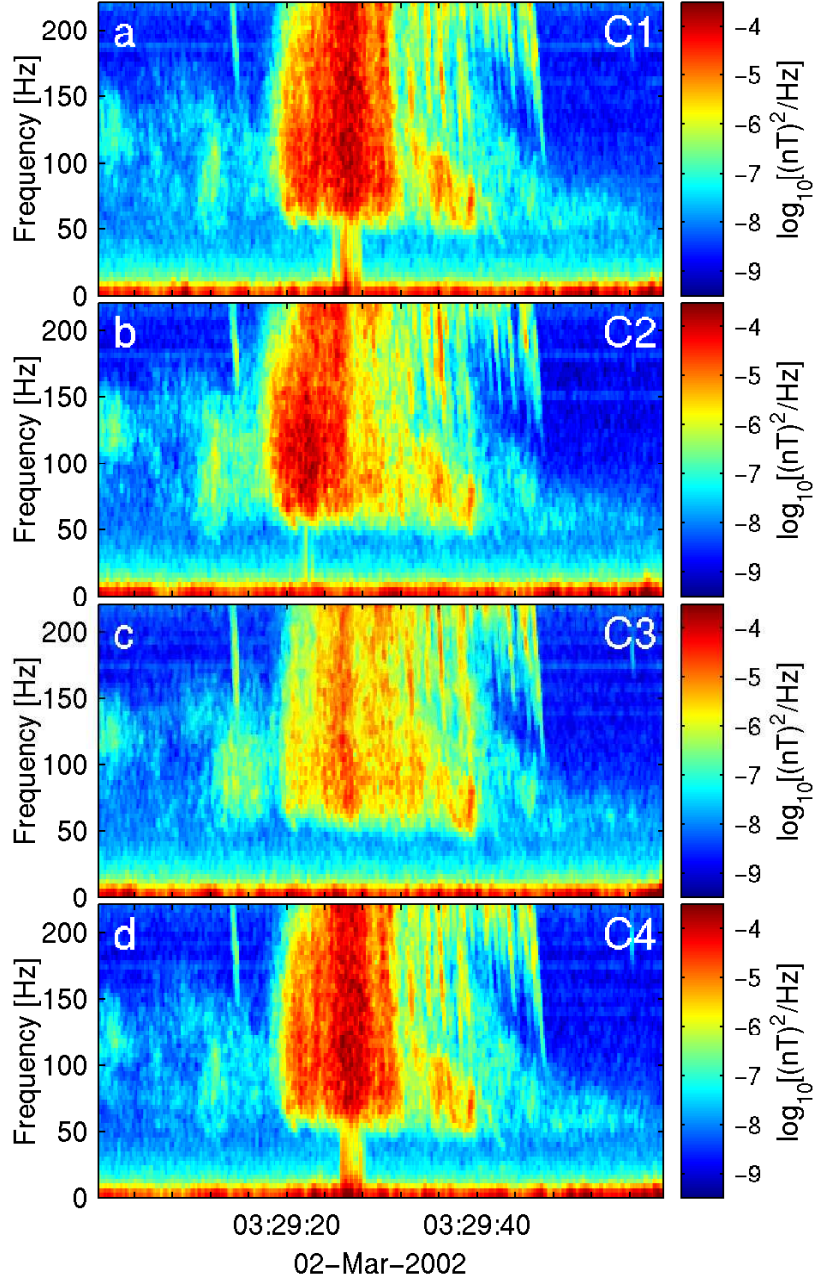

Fig. 3. Frequency-time spectrograms (computed using the total magnetic wave field from STAFF) for all spacecraft. Note the lowfrequency activity (visible on $\mathrm{C} 1, \mathrm{C} 2$ and $\mathrm{C} 4$ ) at the times of most intense waves.

bottom panel of Fig. 2 shows $E / B$, which provides a good estimate of the phase velocity in the case of whistlers, since the wave electric field is perpendicular to the wave vector. We find that the phase velocity is of the order of $5 \cdot 10^{6} \mathrm{~m} / \mathrm{s}$.

\section{Discovering the thin sheets}

All four Cluster satellites observe the wave emission. Frequency-time spectrograms (computed using the total magnetic wave field from STAFF) for all spacecraft are presented in Fig. 3. The emission looks similar on all four spacecraft, although not identical. Of special interest are the low-frequency signatures $(<50 \mathrm{~Hz})$ clearly seen on Cluster 1 (C1) and Cluster 4 (C4) at about 03:29:26 UT. A similar signature is barely visible on Cluster $2(\mathrm{C} 2)$ at approximately 03:29:22 UT. These low-frequency waves coincide with the most intense part of the emissions. On auroral field lines such low-frequency activity has been found to indicate local

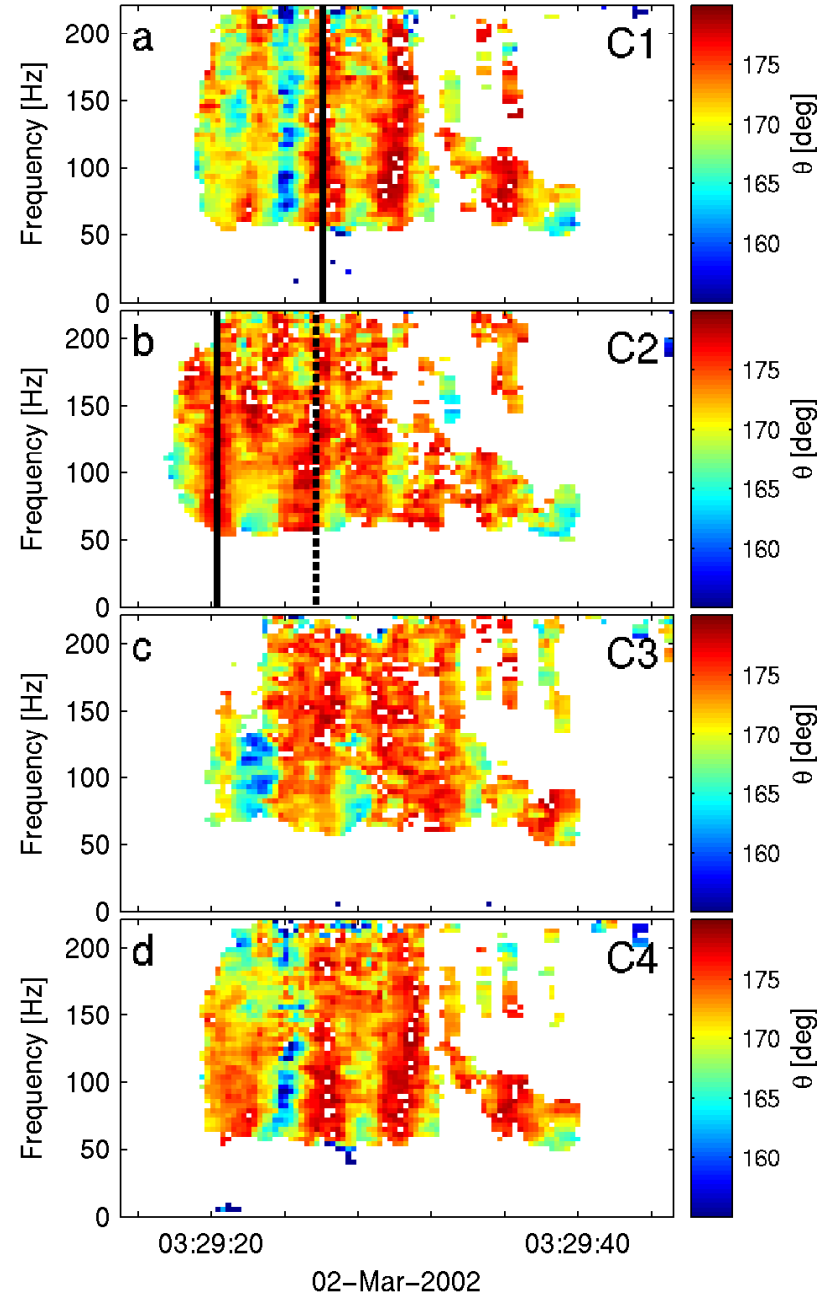

Fig. 4. Distribution of the polar angle, $\theta$, versus time and frequency for $\mathrm{C} 1, \mathrm{C} 2, \mathrm{C} 3$ and $\mathrm{C} 4$. The three vertical lines marking the centers of three stripes observed by $\mathrm{C} 1$ and $\mathrm{C} 2$ are drawn for future reference.

generation of whistler mode waves (James, 1976; Lönnkvist et al., 1993).

An investigation of the propagation direction of the waves makes the interpretation of the low-frequency activity as a marker of a generation region credible. Applying Means' method (Means, 1972) to the magnetic wave field data, we estimate the direction of the wave vector $\boldsymbol{k}$. The analysis does not distinguish between $\boldsymbol{k}$ and $-\boldsymbol{k}$ and therefore we use the result of the Poynting flux calculation from the previous section to remove this ambiguity. All calculations are performed in a background magnetic field oriented coordinate system. The direction of $\boldsymbol{k}$ is specified by the polar angle, $\theta$, (the angle between $\boldsymbol{k}$ and $\boldsymbol{B}_{\mathbf{0}}$ ) and the azimuthal angle, $\phi$, (the angle in the plane perpendicular to $\boldsymbol{B}_{\mathbf{0}}$ ).

Figure 4 presents the polar angle versus time and frequency for all spacecraft. The polar angle $\theta$ is color coded in the range $155^{\circ}<\theta<180^{\circ}$. All time-frequency bins corresponding to a spectral density below $10^{-6}(\mathrm{nT})^{2} / \mathrm{Hz}$ or a 
value of $\theta$ outside the interval given above are removed from the plots. We find that the waves propagate in a roughly $25^{\circ}$ wide cone anti-parallel to $\boldsymbol{B}_{\mathbf{0}}$. Moreover, the angular interval is chosen to enhance the effect of the color coding and a striped pattern appears. For C1, C2 and C4 we identify at least three bands where the waves are more anti-parallel to $\boldsymbol{B}_{\mathbf{0}}$. The three vertical lines in Fig. 4 indicate the centers of three such stripes. The overall structure is similar for all spacecraft, but the stripes are less obvious for Cluster 3 (C3). The low-frequency $(<50 \mathrm{~Hz})$ signatures pointed out earlier in Fig. 3 coincide with the band marked with a solid line in the top (C1) panel and the corresponding structure in the bottom (C4) panel.

A possible interpretation of the banded structure in Fig. 4 is that the emission is composed of three or more fieldaligned, sheet-like structures in which waves are generated. A cross section of such a wave-generating sheet is depicted in Fig. 5, which also illustrates the relation between the magnetopause normal (from Minimum Variance Analysis (Sonnerup and Scheible, 1998)), the plasma drift velocity (from CIS/HIA) and the location of the four Cluster satellites. In the plane perpendicular to $\boldsymbol{B}_{\mathbf{0}}$ (the plane of the figure) the waves propagate out from the sheet. Assuming the sheet is drifting with the plasma velocity, the red arrows in Fig. 5 indicate the waves to be observed before a sheet passes, while the turquoise arrows show the waves to be recorded after a sheet has passed. Hence, from the sketch we realize that the passing of a sheet should result in a sudden shift in the recorded wave vector azimuthal angle $\phi$. In order to investigate if such shifts can be observed, we invoke the remaining part of the results obtained by Means' method. Verification of sudden shifts in direction of propagation would further strengthen the hypothesis of sheet-like structures.

The azimuthal angle, $\phi$, is plotted versus time and frequency in Fig. 6a (C1). To make it easier to interpret the results only two colors are used in the remaining panels: red corresponds to waves preceding a sheet and turquoise to waves succeeding it. All sheets are assumed to be oriented as in Fig. 5. (The direction of the sheets is a free parameter, and the chosen orientation, shown in Fig. 5, is the orientation that best organizes the results of the Means analysis.) An abrupt shift occurring simultaneously on all frequencies is obvious on $\mathrm{C} 1$ and $\mathrm{C} 4$, at the time of the low-frequency wave activity. This is what we expect to see when a wave-generating sheet passes; the direction of propagation changes instantaneously, independent of frequency. Apart from this evident shift, there is also a sudden change in the azimuthal angle coinciding with the third of three bands of anti-parallel waves observed by $\mathrm{C} 1$ and C4 (at 03:29:30 UT). On C2 we see one clear shift (solid line), and we can imagine a less clear shift five seconds later (dashed line), both corresponding to stripes in Fig. 4. Neither of these shifts in propagation direction coincide with the weak low-frequency $(<50 \mathrm{~Hz})$ emission detected by $\mathrm{C} 2$. Instead, the low-frequency waves on $\mathrm{C} 2$ are associated with a less clear band of anti-parallel propagation, which does not result in any visible shift in azimuthal angle. We conclude that all clear shifts in Fig. 6 correspond

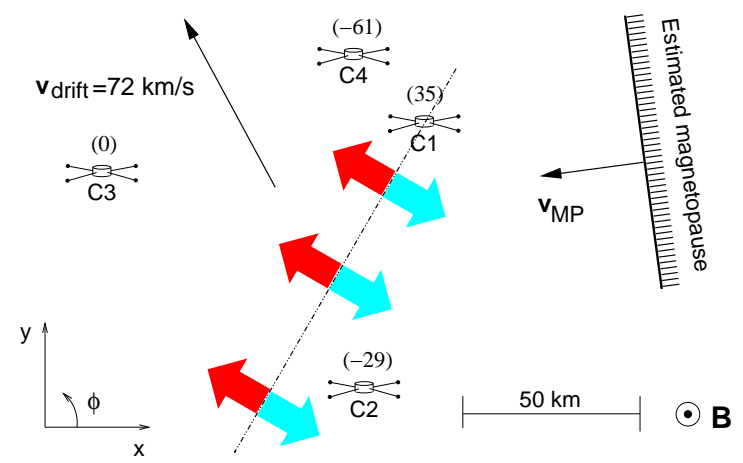

Fig. 5. A suggested thin sheet of wave generation is presented (the dashed-dotted line) in the actual geometry of this event. The location of the spacecraft, as well as the magnetopause normal and plasma drift velocity, is presented in the magnetic field oriented system used throughout the paper. The numbers in brackets are the spacecraft distances in the direction parallel to $\boldsymbol{B}_{\mathbf{0}}$ to the reference satellite $\mathrm{C} 3$.

to stripes found in Fig. 4. These sudden shifts confirm the interpretation of the bands as at least three sheets of waves passing the spacecraft.

Assuming that the sheet-like structures move with the plasma velocity, we arrive at a consistent picture. The ion observations give a drift velocity of $\boldsymbol{v}_{\text {drift }}=(-34,63,5) \mathrm{km} / \mathrm{s}$ (from CIS/HIA on C4) in the magnetic field oriented reference frame depicted in Fig. 5. Hence, we expect a sheet to travel from $\mathrm{C} 2$ to $\mathrm{C} 1$ in about $0.3 \mathrm{~s}$ and from $\mathrm{C} 1$ to $\mathrm{C} 4$ in $0.5 \mathrm{~s}$. Consequently, the less clear shift observed by $\mathrm{C} 2$ (dashed line in Fig. 4) may be caused by the passing of the same sheet responsible for the very distinct change in propagation direction on $\mathrm{C} 1$ (solid line), and $\mathrm{C} 4$ less than a second later. Also, the shift seems to be observed by $\mathrm{C} 1$ fractions of a second before it is detected on C4. All these observations are consistent with a thin sheet of whistlers passing the satellites with the plasma drift velocity.

Since the observations on $\mathrm{C} 1$ and $\mathrm{C} 4$ are very similar the sheet extension parallel to the background field is likely to be much larger than the spacecraft separation of $100 \mathrm{~km}$. The extension of the sheet in the plane perpendicular to $\boldsymbol{B}_{\mathbf{0}}$ is also larger than the spacecraft separation, but there are variations on this scale length since there are differences between the observations made by $\mathrm{C} 1$ and $\mathrm{C} 2$.

Furthermore, if we assume that the low-frequency $(<50 \mathrm{~Hz})$ emission observed by $\mathrm{C} 1$ and $\mathrm{C} 4$ corresponds to a sheet of wave generation moving with the plasma velocity, we can estimate the width of the sheet. If we consider the emission to be two seconds long, the sheet width is about $\Delta L=100 \mathrm{~km}$. This distance is equivalent to $30 \mathrm{c} / \omega_{p e}$ (electron inertial lengths) or $0.75 c / \omega_{p i}$ (ion inertial lengths). However, there are reasons to believe that the sheets, in fact, are even thinner. The very sharp switch in azimuthal angle (cf. Fig. 6a) indicates a narrow sheet and referring to Fig. 3 there are evidence of structures on scale lengths considerably less than $100 \mathrm{~km}$. We conclude that the observed sheets are 


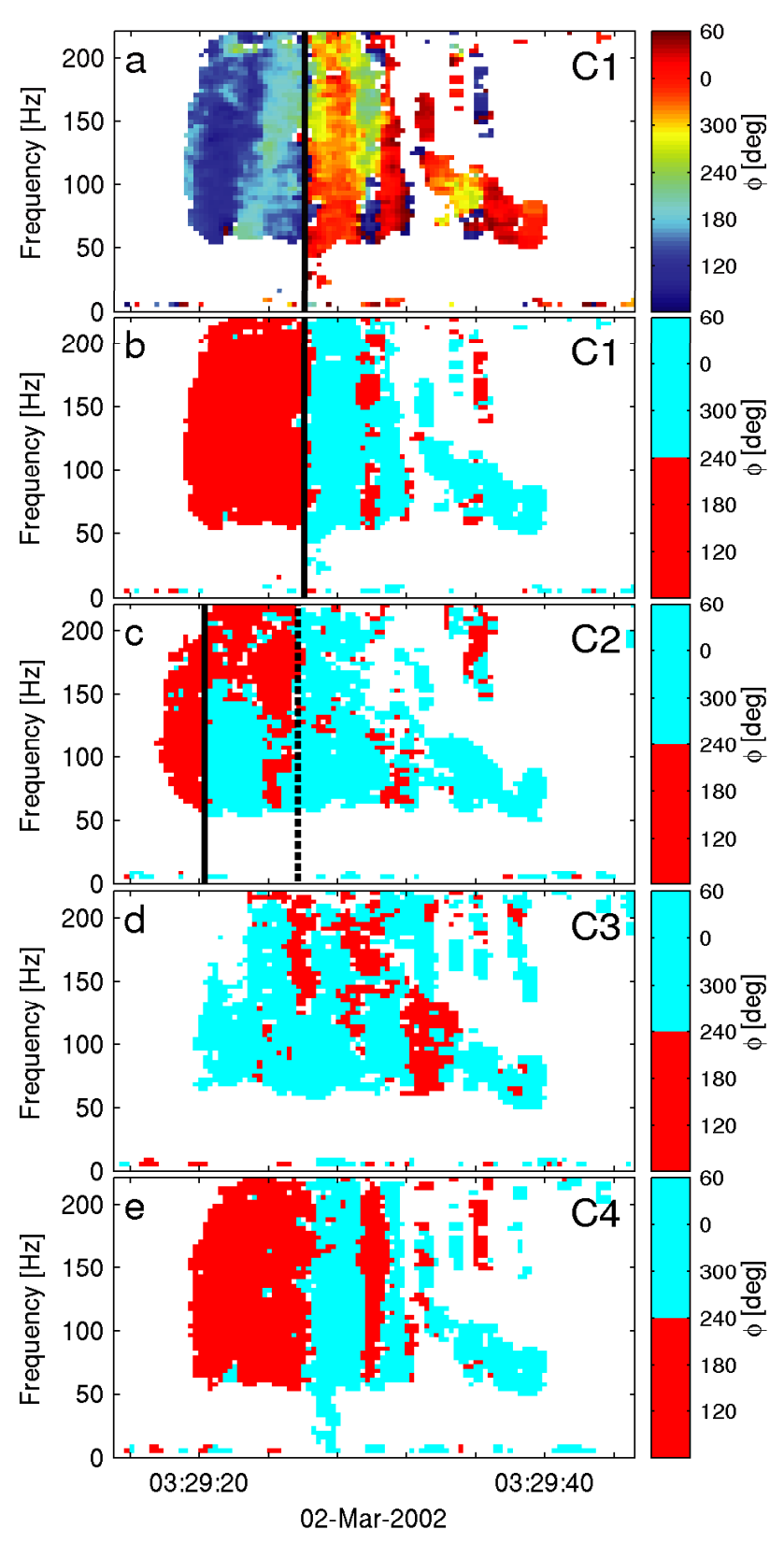

Fig. 6. The azimuthal angle, $\phi$, versus time and frequency for the four spacecraft. The vertical lines in the panels for $\mathrm{C} 1$ and $\mathrm{C} 2$ correspond to the lines in Fig. 4. Hence, the shifts in azimuthal angle coincide with the centers of the bands of more anti-parallel waves.

indeed thin in the meaning that the width is comparable to electron scales.

\section{Correlation and wavelength}

The emissions observed by the different spacecraft are clearly related. Cross-correlating data convincingly demonstrate this relationship and provide estimates of the phase velocity and the wavelength at the same time.
The phase difference of a plane wave registered at two positions in space, $\mathbf{r}_{i}$ and $\mathbf{r}_{j}$, is given by

$\Delta \psi=\mathbf{k} \cdot\left(\mathbf{r}_{i}-\mathbf{r}_{j}\right)=k_{x} d_{i j, x}+k_{y} d_{i j, y}+k_{\|} d_{i j, \|}$,

where $d_{i j}$ are the distances in the different directions between the two positions, for instance, $d_{i j, \|}=\hat{\mathbf{z}} \cdot\left(\mathbf{r}_{i}-\mathbf{r}_{j}\right)$ and $k_{x}, k_{y}$ and $k_{\|}$are the components of the wavevector in the two perpendicular and the parallel directions with the respect to the background magnetic field.

If we assume that the waves propagate mainly along the background magnetic field, $\mathbf{k}=k_{\|} \hat{\mathbf{z}}$, the phase difference of a plane wave registered at two positions, $\mathbf{r}_{i}$ and $\mathbf{r}_{j}$, is given by Holmgren and Kintner (1990)

$\Delta \psi=k_{\|} d_{i j, \|}=\frac{\omega}{v_{z}} d_{i j, \|} \cdot$

To arrive at the final expression we have used the parallel phase velocity, $v_{z}$, given by $\omega=v_{z} k_{\|}$.

To examine the phase differences $(\Delta \psi)$ we compute the cross spectra between the $x$-components of the wave magnetic field observed by the different spacecraft. Figure 7 presents the cross spectral densities, $S_{B x B x^{\prime}}$, plotted versus phase and frequency. The phase of the cross spectrum, $\eta_{x y}$, is equivalent to the averaged phase difference, $\langle\Delta \psi\rangle$, between the two recorded signals, up to an uncertainty of $2 \pi$. Only correlations with $\mathrm{C} 1$ are shown since the phase difference between, for example, $\mathrm{C} 2$ and $\mathrm{C} 3$, can be derived from the correlations between $\mathrm{C} 1$ and $\mathrm{C} 2$ and $\mathrm{C} 1$ and $\mathrm{C} 3$, respectively. The coherences in the frequency range of interest are about $0.5,0.5$ and 0.9 , respectively, and confirm a strong correlation. The correlation between satellites $\mathrm{C} 1$ and $\mathrm{C} 4$ is most obvious, which is expected since these spacecraft are almost on the same field lines.

To estimate the phase velocity at $100 \mathrm{~Hz}$ we use Eq. (2) and the observed $\langle\Delta \psi\rangle$ to compute $v_{z}$ for a few different multiples of $2 \pi$. The calculations are indicated in Fig. 7. All spacecraft agree only for an estimated phase velocity of $5.4-5.9 \cdot 10^{3} \mathrm{~km} / \mathrm{s}$ anti-parallel to the ambient magnetic field. The related wavelength is about $50 \mathrm{~km}$. The phase velocity obtained in this way is very close to the estimate found using $E / B\left(5 \cdot 10^{3} \mathrm{~km} / \mathrm{s}\right.$, cf. Fig. 2$)$.

The group velocity can be found noting that

$\frac{d\langle\Delta \psi\rangle}{d \omega}=\frac{d k_{\|}}{d \omega} d_{i j, \|}=\frac{d_{i j, \|}}{v_{g}}$,

where $v_{g}$ is the group velocity, assuming parallel propagation. The correlation between $\mathrm{C} 1$ and $\mathrm{C} 4$ gives the most clearly defined slope, and we use this satellite pair to estimate $v_{g}$. We find $v_{g} \approx-10000 \mathrm{~km} / \mathrm{s}$, which is about twice the phase velocity. This fits nicely with a quadratic dispersion, $\omega=C \cdot k^{2}$, which is expected for whistlers in this frequency range.

An alternative way to determine the wave vector (propagation direction and wavelength) is the wave distribution function (WDF) analysis (Storey and Lefeuvre, 1974; Storey and Lefeuvre, 1979; Storey and Lefeuvre, 1980; Oscarsson and 

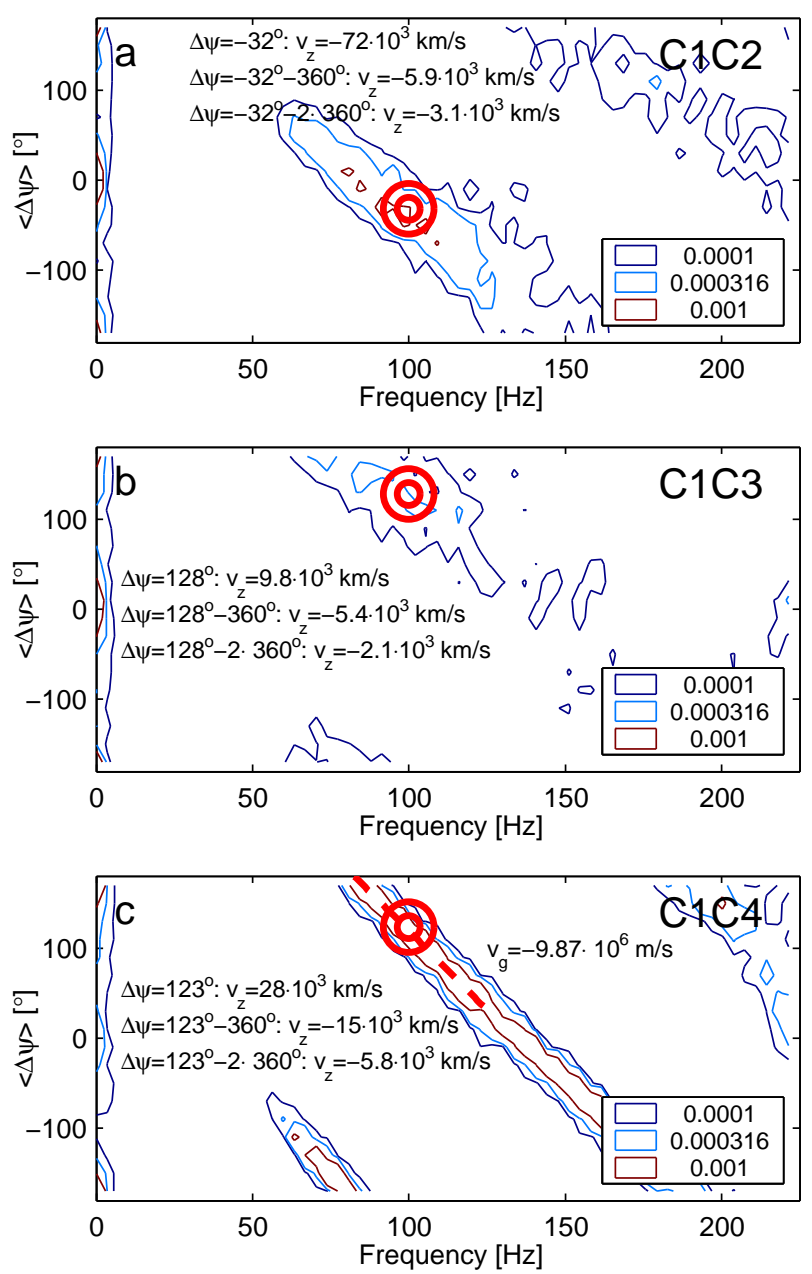

Fig. 7. The contours show the distribution of cross spectral density, $S_{B x B x^{\prime}}$, in frequency, $f$, and averaged phase difference, $\langle\Delta \psi\rangle$ $\left(=\eta_{x y}\right)$. Data used are from the STAFF experiment. The cross spectral density is in units of $(\mathrm{nT})^{2} /{ }^{\circ} \mathrm{Hz}$. We have used a record length of 256 points and have averaged over 101 time records and two frequencies. The total time interval used is $60 \mathrm{~s}$, starting from 03:29:00 UT. The top panel shows the result from correlating signals from $\mathrm{C} 1$ and $\mathrm{C} 2$, the middle panel displays the result from correlating $\mathrm{C} 1$ and $\mathrm{C} 3$, and the bottom panel is produced correlating $\mathrm{C} 1$ and $\mathrm{C} 4$. The coherences in the frequency range of interest are about $0.5,0.5$ and 0.9 , respectively. The phase velocity, $v_{z}$, is estimated at $100 \mathrm{~Hz}$. A few possible values of $v_{z}$, assuming a different number of multiples of $2 \pi$, are indicated in each panel. All spacecraft pairs agree for a phase velocity of about $5.4-5.9 \cdot 10^{3} \mathrm{~km} / \mathrm{s}$ anti-parallel to $\mathbf{B}_{\mathbf{0}}$. The group velocity is determined only for the $\mathrm{C} 1 / \mathrm{C} 4$ case. It is inversely proportional to the slope of a straight line fitted to the results and the estimate yield about $10000 \mathrm{~km} / \mathrm{s}$.

Rönnmark, 1989). The idea of WDF-analysis is to use all available polarization information in the data to reconstruct the wave energy distribution in wave vector space. To this end, a description of the plasma is required and Table 1 summarizes the plasma model used. We assume a quasi-neutral proton-electron plasma, with a plasma density of $3.0 \mathrm{~cm}^{-3}$ (from WHISPER). The waves are observed in a boundary
Table 1. The plasma model. All components are assumed to be Maxwellians. The model is based on PEACE and CIS observations.

\begin{tabular}{llll}
\hline Species & Density $\left(\mathrm{cm}^{-3}\right)$ & $T_{\|}(\mathrm{eV})$ & $T_{\perp}(\mathrm{eV})$ \\
\hline $\mathrm{H}^{+}$ & 3.0 & 2000 & 2000 \\
$\mathrm{e}^{-}$ & 2.6 & 40 & 10 \\
$\mathrm{e}^{-}$ & 0.4 & 400 & 400 \\
\hline
\end{tabular}

layer with two different electron components: The magnetospheric high energy electrons are modelled as an isotropic Maxwellian with a thermal energy of $400 \mathrm{eV}$. The low energy electrons originating from the magnetosheath are assumed to be anisotropic with a parallel thermal energy of $40 \mathrm{eV}$ and $T_{\|} / T_{\perp}=4$ (cf. Sect. 7). The protons (not shown in this paper) are regarded as isotropic, with a thermal energy of $2 \mathrm{keV}$.

The computer code WHAMP (Rönnmark, 1982) is used to solve the dispersion relation for linear waves in the plasma described in Table 1. At a given frequency an existing wave mode appears as a curve in wave vector space (cf. Fig. 8). Along such a constant frequency contour the polarization varies. Hence, the polarization information contained in the observed wave fields (phase and amplitude relations between the different wave field components) can be used to localize the wave energy on this curve in k-space. Reconstructing WDF is doing that in a systematic way.

The WDF is related to the measured data through the spectral matrix. Given a distribution of wave energy in wave vector space, all spectral densities can be computed. The inverse problem, however, is underdetermined and we use a maximum entropy method to select a unique WDF, given an observed spectral matrix. The reader is referred to Oscarsson (1994) for details of the algorithm and to Stenberg et al. (2002) for an application.

While WDF-analysis assumes a homogeneous plasma, the event we study is highly structured in the plane perpendicular to $\boldsymbol{B}_{\mathbf{0}}$. We still believe that the approximations made are useful since the waves propagate mainly parallel to $\boldsymbol{B}_{\mathbf{0}}$, and the parallel scale length of the thin sheets is most likely much larger than the parallel wave length obtained $(\approx 50 \mathrm{~km})$.

The result from a WDF-analysis is shown in Fig. 8. We have reconstructed the WDF on $100 \mathrm{~Hz}$, using $2 \mathrm{~s}$ of data from C4 (03:29:20.5-03:29:22.5 UT). The top panel shows the constant frequency contour in wave vector space. The circle indicates the location of the peak in the WDF. The second and third panels show the WDF versus (normalized) $k_{\perp}$ and azimuthal angle $(\phi)$, respectively.

In short, the WDF-analysis is consistent with the Means analysis: The angle between $\mathbf{k}$ and $\mathbf{B}$ is $170-175^{\circ}$ and the azimuthal angle $\phi=100^{\circ}$ (compare Fig. 4 and Fig. 6). This gives credibility to both the WDF and the Means analysis. The reconstructed WDF in Fig. 8 peaks at $k_{\|} \rho_{H^{+}}=25$, giving a parallel wavelength of about $50 \mathrm{~km}$. Hence, reconstruction of the WDF and correlation analysis independently give roughly the same parallel wavelength. 

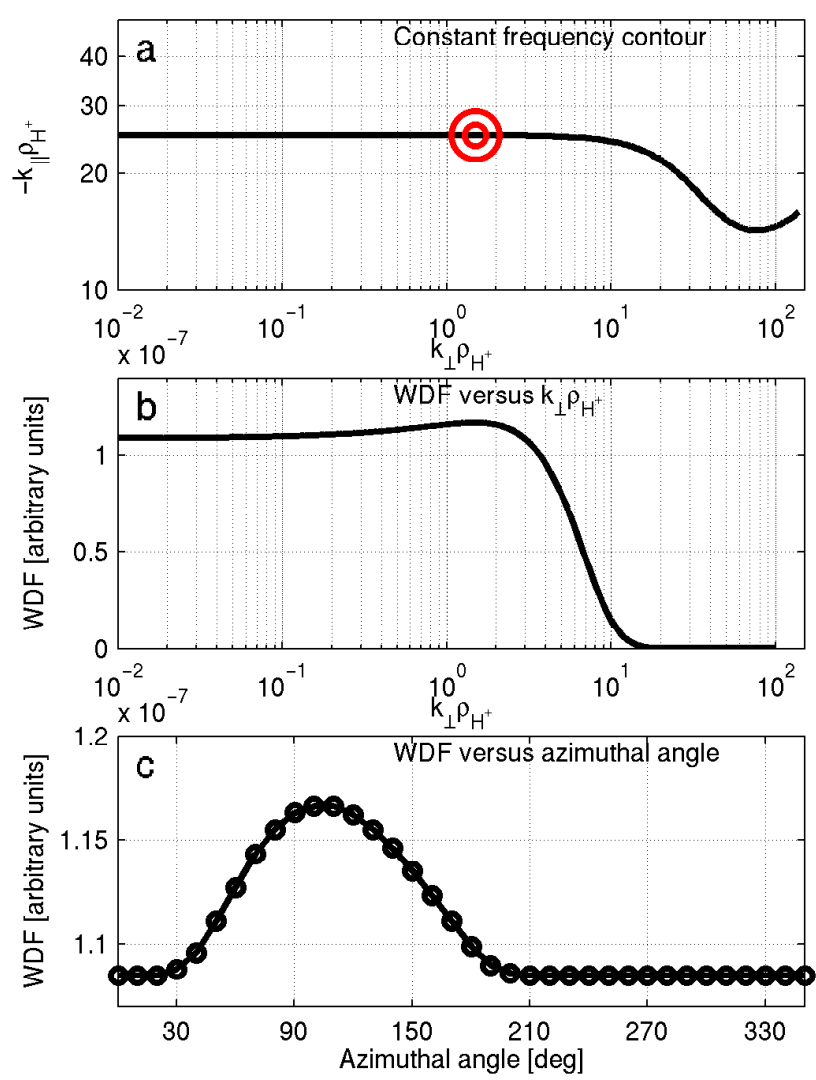

Fig. 8. (a) Constant frequency contour for the whistler mode at $100 \mathrm{~Hz}$ in $\boldsymbol{k}$-space. The wave vectors are normalized to the gyroradius of the $2 \mathrm{keV}$ protons in the model, that is, $\rho_{H^{+}}=208 \mathrm{~km}$. The parallel wave vectors are negative, which correspond to propagation anti-parallel to $\boldsymbol{B}_{\mathbf{0}}$. The peak of the reconstructed WDF is indicated. (b) WDF versus $k_{\perp} \rho_{H^{+}}$. (c) WDF versus azimuthal angle $\phi$.

\section{Electron signatures and connections to the magne- topause}

Although Cluster data reveal that whistler waves frequently exist in the vicinity of the magnetopause, proof of a direct link to the boundary is missing. However, electron observations provide additional information and invite to speculations on a coupling.

As pointed out earlier, the whistler wave emission is observed in a boundary layer where magnetospheric and magnetosheath plasma coexist. Figure 9 presents electron flux versus time and energy for C4. Different panels correspond to different pitch angle intervals. Prior to 03:31:10 UT, when $\mathrm{C} 4$ crosses the magnetopause, there is a mixture of hot magnetospheric and cold magnetosheath electrons.

At the time when the whistler wave generation region passes the spacecraft, an intriguing feature is seen in the electrons: The magnetospheric $(400 \mathrm{eV})$ particles almost seem to vanish. This signature is marked by arrows in Fig. 9. It is most clearly seen in the second panel, but it is visible in all three middle panels. Parallel and anti-parallel to the background magnetic field there is no apparent change in electron

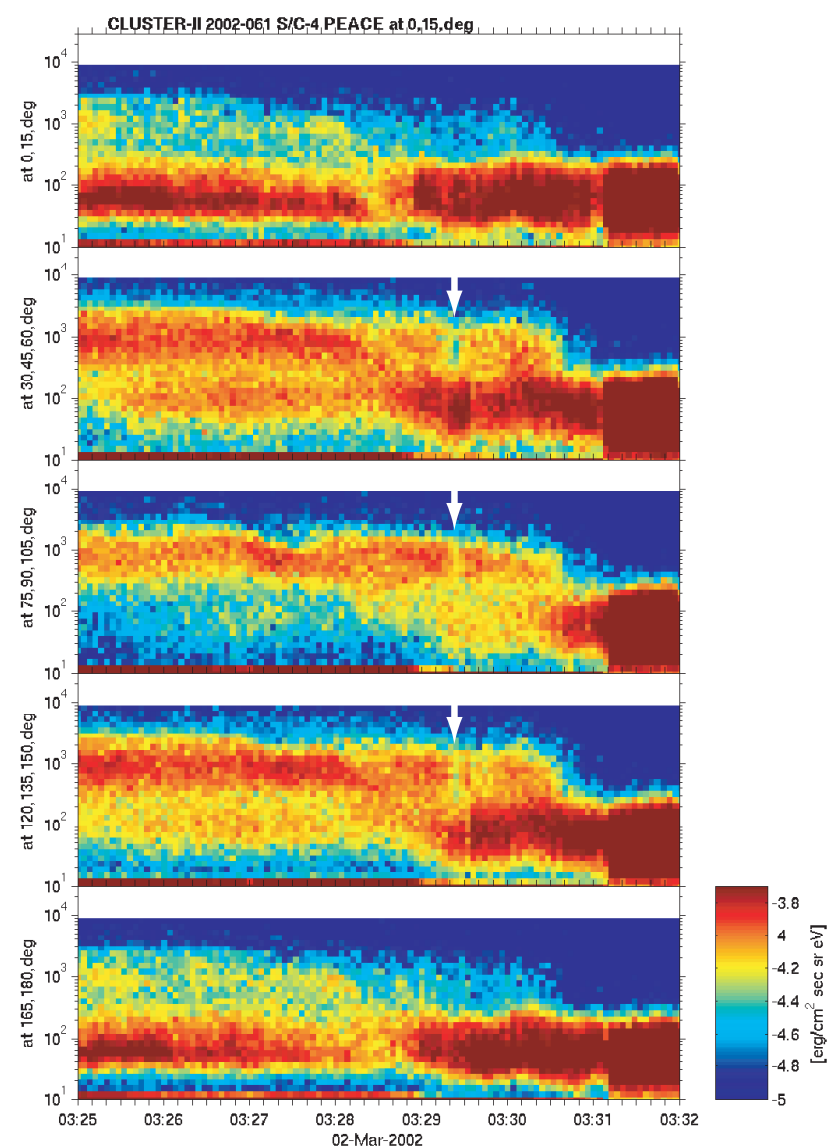

Fig. 9. Electron flux versus time and energy for $\mathrm{C} 4$. The sudden decrease in the flux of high energy $(400 \mathrm{eV})$ particles (indicated by arrows) coincides with the most intense waves.

flux, but in these directions the flux of the high energy particles is already low. The electron flux observed by $\mathrm{C} 1$ is very similar (not shown). A sudden decrease in the high energy electrons is also detected by $\mathrm{C} 2$ (not shown), but requires some imagination to identify. In $\mathrm{C} 3$ data no such signature can be recognized. Due to the limited time resolution of the PEACE instrument, a time shift between $\mathrm{C} 2$ and $\mathrm{C} 1 / \mathrm{C} 4$, in accordance with the observations of the low frequency signatures (cf. Fig. 3), is difficult to verify.

The disappearance of the magnetospheric electrons itself, however, allows for an interesting interpretation. Suppose the field lines in question are or have very recently been attached to a reconnection site. Then the high energy particles can escape through the "hole" in the magnetopause, which the diffusion region constitutes. If this is the case, these field lines are directly coupled to processes at the magnetopause and provide the connection between the magnetopause dynamics and the observed whistler waves.

\section{Generating the whistler waves}

To further establish the link between the observed waves and the magnetopause, we investigate which parameters govern 

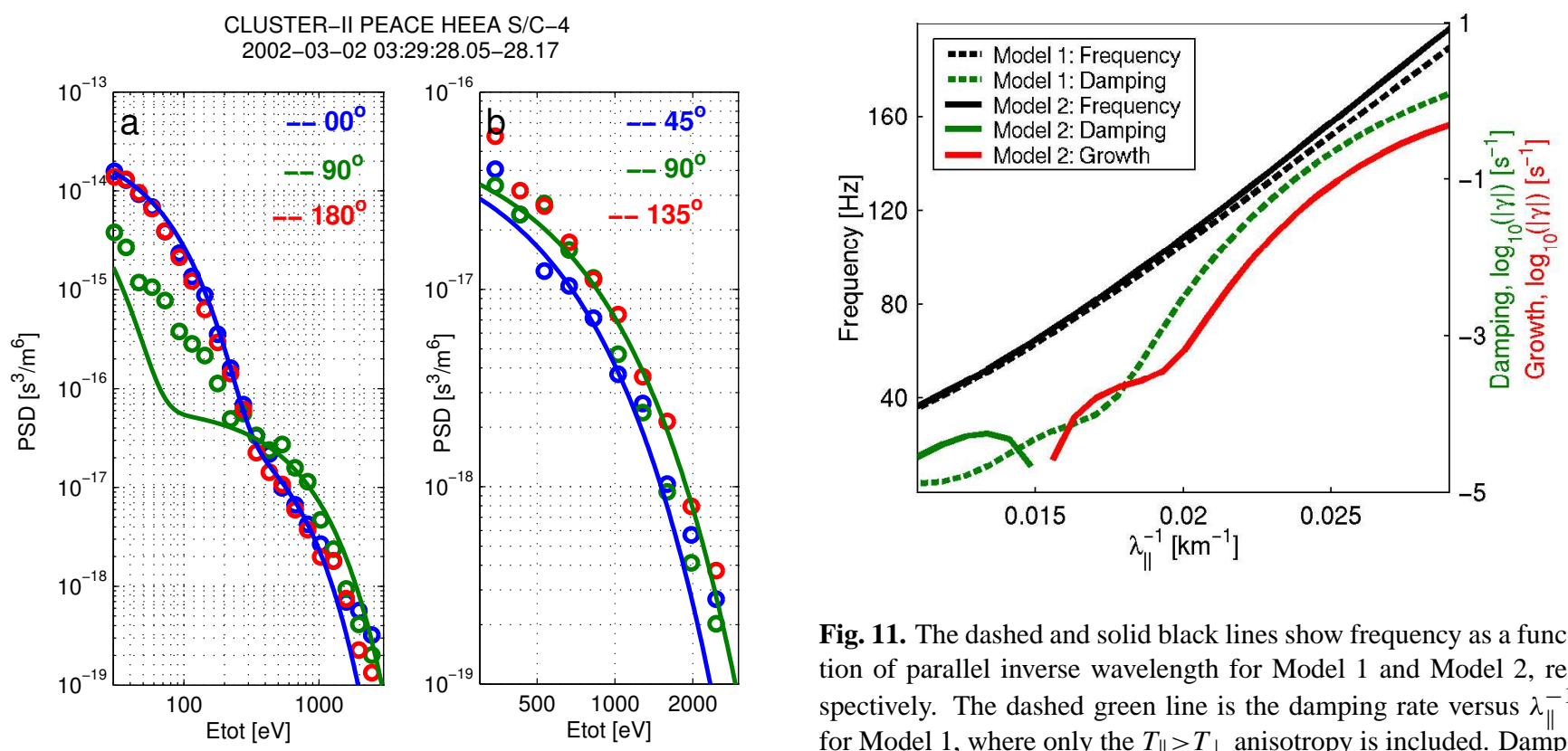

Fig. 10. Cross sections through the electron distribution function. (a) The phase space density of electrons for angles parallel, antiparallel and perpendicular to the magnetic field. We see that for electron energies ranging from $20-200 \mathrm{eV}$, the phase space density is clearly larger parallel/anti-parallel than perpendicular to the magnetic field, whereas the opposite is true for energies of $200 \mathrm{eV}$ $1 \mathrm{keV}$. The circles are observations while solid lines show the model used. (b) Phase space density of high energy electrons for pitch angles $45^{\circ}, 90^{\circ}$ and $135^{\circ}$. Data (circles) clearly shows that the phase space density is lower nearly parallel $\left(45^{\circ}\right)$ than nearly anti-parallel $\left(135^{\circ}\right)$. The model electron distributions for $90^{\circ}$ and $45 / 135^{\circ}$ are included (solid lines).

Table 2. The second plasma model. All components are assumed to be Maxwellians. The model is based on PEACE and CIS observations. Compared to Model 1, we assume slightly less cold electrons and introduce an inverse temperature anisotropy at higher energies.

\begin{tabular}{llll}
\hline Species & Density $\left(\mathrm{cm}^{-3}\right)$ & $T_{\|}(\mathrm{eV})$ & $T_{\perp}(\mathrm{eV})$ \\
\hline $\mathrm{H}^{+}$ & 3.0 & 2000 & 2000 \\
$\mathrm{e}^{-}$ & 2.4 & 40 & 10 \\
$\mathrm{e}^{-}$ & 0.6 & 400 & 600 \\
\hline
\end{tabular}

the wave generation. Several different generation mechanisms have been suggested to generate whistler mode waves in the bowshock/magnetopause region (Tsurutani and Rodriguez, 1981; Tokar et al., 1984; Veltri and Zimbardo, 1993; Sakai et al., 2000), all involving anisotropic electrons as the free energy source.

The current case does not lack sources of free energy. Figure 10a shows cross sections through the electron distribution function at three different pitch angles (colored circles). For electron energies $20-200 \mathrm{eV}$ the phase space density is larger parallel/anti-parallel to the background mag-

netic field than perpendicular to it. The opposite is true at higher energies $(200 \mathrm{eV}-1 \mathrm{keV})$. Moreover, occasionally during the wave emission we record an enhanced phase space density at oblique angles (electron conics) between $20-40 \mathrm{eV}$ (not shown).

In principle, both the temperature anisotropies can be responsible for wave growth. However, referring to Gary (1993), we expect that in our case, with a very low plasma $\beta$ (the ratio between the plasma and magnetic pressures), only the $T_{\perp}>T_{\|}$instability will, in fact, work. To confirm this assumption we compare the stability properties of two different models using the WHAMP code. The first model was presented and used in Sect. 6 and takes into account only the anisotropy at lower energies. Table 2 shows the second model, where the anisotropy at larger energies are also considered. The modelled electron distribution is also included in Fig. 10a for comparison with the observations.

The results from the WHAMP analysis are summarized in Fig. 11. The solid and dashed black lines display the (real part of the) frequency as a function of $\lambda_{\|}^{-1}$ for the two models. Only minor differences are seen. In the WDF analysis performed at $100 \mathrm{~Hz}$, in Sect. 6, only the real part is considered and, hence, the results obtained will be virtually the same regardless of which one of the two models is used.

The growth/damping properties are, on the other hand, very different for the two cases. For Model 1 the waves are damped throughout the frequency range of interest (green dashed line), whereas for Model 2 the waves grow for frequencies ranging from $60-300 \mathrm{~Hz}$ (red solid line). This is in almost perfect agreement with observations, where whistler waves are observed from $60 \mathrm{~Hz}$ up to about $400 \mathrm{~Hz}$. The possible wave growth due to electron conics has also been tested, 
modelling the conic using a loss-cone distribution. We find that such a model is not able to generate wave growth in a broad frequency range for reasonable plasma parameters.

Model 2 is symmetric with respect to parallel and antiparallel propagation. To explain why we detect only antiparallel propagating waves, we need to consider the electron distribution in closer detail. Figure 10b shows the high energy part of the distribution for three different pitch angles, $45^{\circ}, 90^{\circ}$ and $135^{\circ}$. It is clear that the phase space density is lower at $45^{\circ}$ (blue circles) than at $90^{\circ}$ and $135^{\circ}$ (green and red circles), for the energies shown. The same tendency can also be seen in Fig. 9.

To fulfill the resonance conditition, the generated waves must propagate in the opposite direction of the electron anisotropy responsible for their growth. Thus, as the anisotropy is considerably larger nearly parallel to the field than nearly anti-parallel, the waves should propagate antiparallel (away from Earth), as is observed. This observation is also consistent with the speculation that the high energy electrons are lost through a "hole" in the magnetopause. In that case the parallel and nearly parallel electrons would be most affected.

Future detailed studies of the whistler waves must also consider the simultaneously detected high frequency waves $(5-25 \mathrm{kHz})$. These waves occur at the same time as the whistlers and hence the propagation properties and/or generation/damping processes must be related. Simultaneous whistlers and electron plasma oscillations have previously been observed in the solar wind by Kennel et al. (1980).

\section{Summary of conclusions}

This paper is an event study of whistler mode waves observed by the Cluster satellites on the magnetospheric side of the magnetopause. The main conclusions are summarized below.

- Whistler mode waves in the vicinity of the magnetopause are generated in thin sheets. The Cluster satellites record a passage of such sheets moving with the plasma drift velocity. The sheet width is estimated to be less than $30 c / \omega_{p e}$ (less than an ion inertial length), proving that small-scale physics is important in the magnetopause region, even at large distances from reconnection sites.

- The whistler waves propagate anti-parallel to the ambient field (away from Earth towards the magnetopause), with an angle between $\boldsymbol{k}$ and $\boldsymbol{B}_{\mathbf{0}}$ in the interval 0-25. The parallel wavelength is found to be of the order of $50 \mathrm{~km}$ at $100 \mathrm{~Hz}$.

- A sudden disappearance of magnetospheric electrons is interpreted as evidence of field lines that are or have been connected to a reconnection site. The decrease in electron flux coincides with the time of wave generation. This provides a direct coupling between the observed waves and processes at the magnetopause itself.
- The anisotropic electrons observed in the whistler wave region consitute the free energy needed to generate the waves. The temperature anisotropy $\left(T_{\perp}>T_{\|}\right)$observed at high energies $(300 \mathrm{eV}-1 \mathrm{keV})$ results in an instability that agrees well with the detected emission with respect to the frequency range. Also, the observed anisotropy is significantly larger parallel to the ambient field than anti-parallel, consistent with picturing a "hole" in the magnetopause through which hot electrons are lost. The particles coming from the magnetopause (parallel to the magnetic field) are affected the most and the resulting waves are observed to propagate anti-parallel to the ambient field, as we expect.

- Although some of the fine-structure in this study is visible in the particle data, wave observations are a more sensitive tool to investigate the smallest scale lengths. Wave data reveal structures finer than can be resolved by the particle instruments.

Acknowledgements. We are grateful to the FGM team (A. Balogh) for supplying data for this study. We also acknowledge P. Robert for help with the STAFF magnetic wave field data.

Topical Editor T. Pulkkinen thanks X. Deng and another referee for their help in evaluating this paper.

\section{References}

André, M., Vaivads, A., Buchert, S. C., et al.: Thin electronscale layers at the magnetopause, Geophys. Res. Lett., 31, doi:10.1029/2003GL018137, 2004.

Balogh, A., Dunlop, M. W., Cowley, S. W. H., et al.: The Cluster Magnetic Field Investigation, Space Sci. Rev., 79, 65-91, 1997.

Baumjohann, W., Georgescu, E., Fornacon, K.-H., Auster, H. U., Treumann, R. A., and Haerendel, G.: Magnetospheric lion roars, Ann. Geophys., 18, 406-410, 2000,

SRef-ID: 1432-0576/ag/2000-18-406.

Cattell C., Wygant, J., Mozer, F. S., et al.: ISEE 1 and Geotail observations of low-frequency waves at the magnetopause, J. Geophys. Res, 100, 11 823-11829, 1995.

Cornilleau-Wehrlin, N., Chauveau, P., Louis, S., et al.: The Cluster spatio-temporal analysis of field fluctuations (STAFF) experiment, Space Sci. Rev., 79, 107-136, 1997.

Cornilleau-Wehrlin, N., Chanteur, G., Perraut, S., et al.: First results obtained by the Cluster STAFF experiment, Ann. Geophys., 21, 437-456, 2003,

SRef-ID: 1432-0576/ag/2003-21-437.

Deng, X. H. and Matsumoto, H.: Rapid magnetic reconnection in the Earth's magnetosphere mediated by whistler waves, Nature, 410, 557-560, 2001.

Décréau, P. M. E., Fergeau, P., Krannosels'kikh, M., et al.: WHISPER, a resonance sounder and wave analyser: Performance and perspectives for the Cluster mission, Space Sci. Rev., 79, 157193, 1997.

Escoubet, C. P., Schmidt, R., and Goldstein, M.: Cluster - Science and Mission Overview, Space Sci. Rev., 79, 11-32, 1997.

Escoubet, C. P., Fehringer, M., and Goldstein, M.: The Cluster Mission, Ann. Geophys., 19, 1197-1200, 2001,

SRef-ID: 1432-0576/ag/2001-19-1197. 
Gary, S. P.: Theory of Space Plasma Microinstabilities, Cambridge University Press, 135-139, 1993.

Gurnett D. A., Anderson, R. R., Tsurutani, B. T., et al.: Plasma Wave Turbulence at the Magnetopause: Observations From ISEE 1 and 2, J. Geophys. Res., 84, 7043-7058, 1979.

Gustafsson, G., Boström, R., Holback, B., et al.: The electric field and wave experiment for the Cluster mission, Space Sci. Rev., 79, 137-156, 1997.

Holmgren, G. and Kintner, P.: Experimental Evidence of Widespread Regions of Small-Scale Plasma Irregularities in the Magnetosphere, J. Geophys. Res., 95, 6015-6023, 1990.

LaBelle J. and Treumann, R. A.: Plasma waves at the dayside magnetopause, Space Sci. Rev., 47, 175-202, 1988.

Lönnqvist, H., André, M., Matson, L., et al.: Generation of VLF saucer emissions observed by the Viking satellite, J. Geophys. Res., 98, 13 564-13 574, 1993.

James, H. G.: VLF saucers, J. Geophys. Res., 81, 501-515, 1976.

Johnstone, A. D., Alsop, C., Burge, S., et al.: PEACE: A plasma electron and current experiment, Space Sci. Rev., 79, 351-398, 1997.

Kennel, C. F., Scarf, F. L., Coroniti, F. V., et al.: Correlated Whistler and Electron Plasma Oscillation Bursts Detected on ISEE-3, Geophys. Res. Lett., 7, 129-132, 1980.

Maksimovic, M., Harvey, C. C., Santolik, O., et al.: Polarization and propagation of lion roars in the dusk side magnetosheath, Ann. Geophys., 19, 1429-1438, 2001,

SRef-ID: 1432-0576/ag/2001-19-1429.

Means, J. D.: Use of the Three-Dimensional Covariance Matrix in Analyzing the Polarization Properties of Plane Waves, J. Geophys. Res., 77, 5551-5559, 1972.

Øieroset, M.: In situ detection of collisionless reconnection in the Earth's magnetotail, Nature, 412, 414-417, 2001.

Olson, J. V, Hozer, R. E., and Smith, E. J.: High-Frequency Magnetic Fluctuations Associated with the Earth's Bow Shock, J. Geophys. Res., 74, 4601-4617, 1969.

Oscarsson, T.: Dual Principles in Maximum Entropy Reconstruction of the Wave Distribution Function, J. Comput. Phys., 110, 221-233, 1994.

Oscarsson, T. and Rönnmark, K.: Reconstruction of wave distribution functions in warm plasmas, J. Geophys. Res., 94, 24172427, 1989.

Paschmann, G.: Plasma acceleration at the Earth's magnetopause: Evidence for reconnection, Nature, 282, 243-246, 1979.

Pedersen, A., Décréau, P., Escoubet, C.-P., et al.: Four-point high time resolution information on electron densities by the electric field experiment (EFW) on Cluster., Ann. Geophys., 19, 14831489, 2001,

SRef-ID: 1432-0576/ag/2001-19-1483.

Petkaki, P. C., Watt, E. J., Home R. B., et al.: Anomalous resistivity in non-Maxwellian plasmas, J. Geophys. Res., 108, doi:10.029/2003JA010092, 2003.

Pickett, J., Menietti, J. D., Dowell, J. H., et al.: Polar spacecraft observations of the turbulant outer cusp/magnetopause boundary layer of Earth, Nonlin. Processes Geophys., 6, 195-204, 1999,

SRef-ID: 1607-7946/npg/1999-6-195.

Rème, H., Bosqued, J. A., Sauvaud, J. A., et al.: The Cluster Ion Spectrometry (CIS) Experiment, Space Sci. Rev., 79, 303-350, 1997.

Rezeau, L. and Belmont, G.: Magnetic turbulence at the magnetopause, a key problem for understanding the solar wind/magnetosphere exchanges, Space Sci. Rev., 95, 427-441, 2001 .
Rodriguez, P. and Gurnett D. A.: Electrostatic and Electromagnetic Turbulence Associated With the Earth's Bow Shock, J. Geophys. Res., 80, 19-31, 1975.

Rönnmark, K.: WHAMP - Waves in Homogeneous Anisotropic Multicomponent Plasmas, KGI Report 179, Kiruna Geophysical Institute, 1982.

Sakai, J. I., Nakayama, T., Nambu, M., et al.: Emission of whistler and electromagnetic waves from an electron shear-flow instability, Phys. Lett. A, 265, 103-110, 2000.

Scholer, M., Sidorenko, I., and Jaroschek, C. H.: Onset of collisionless magnetic reconnection in thin current sheets: Threedimensional particle simulations, Phys. Plasmas, 10, 3521-3527, 2003.

Smith E. J., Holzer, R. E., McLeod, M. G., et al.: Magnetic Noise in the Magnetosheath in the Frequency Range 3-300 Hz, J. Geophys. Res., 72, 4803-4813, 1967.

Sonnerup, B. U. Ö. and Scheible, M.: Minimum and Maximum Variance Analysis, Analysis Methods for Multi-Spacecraft Data, ISSI Scientific Report SR-001, 185-220, 1998.

Stenberg, G., Oscarsson, T., André, M., et al.: Investigating wave data from the FAST satellite by reconstructing the wave distribution function, J. Geophys. Res., 107, doi:10.1029/2001/2001JA900154, 2002.

Storey, L. R. O. and Lefeuvre, F.: Theory for the interpretation of measurements of the six components of a random electromagnetic wave field in space, Space Res., 14, 381-386, 1974.

Storey, L. R. O. and Lefeuvre, F.: The analysis of 6-component measurements of a random electromagnetic wave field in a magnetoplasma, I, The direct problem, Geophys. J. R. Astron. Soc., 56, 255-269, 1979.

Storey, L. R. O. and Lefeuvre, F.: The analysis of 6-component measurements of a random electromagnetic wave field in a magnetoplasma, II, The integration kernels, Geophys. J. R. Astron. Soc., 62, 173-194, 1980.

Tokar, R. L., Gurnett, D. A., and Feldman, W. C.: Whistler Mode Turbulence Generated by Electron Beams in Earth's Bow Shock, J. Geophys. Res., 89, 105-114, 1984.

Tsurutani, B. T. and Rodriguez, P.: Upstream Waves and Particles: An Overview of ISEE Results, J. Geophys. Res, 86, 4319-4324, 1981.

Vaivads, A., André, M., Buchert, S. C., et al.: Cluster observations of lower hybrid turbulence within thin layers at the magnetopause, Geophys. Res. Lett., 31, doi:10.1029/2003GL018142, 2004a.

Vaivads, A., Khotyaintsev, Y., André, M., et al.: Structure of the magnetic reconnection diffusion region from four-spacecraft observations, Phys. Rev. Lett., 93, doi:10.1103/PhysRevLett.93.105001, 2004b.

Veltri, P. and Zimbardo, G.: Electron-whistler interaction at the Earth's bow shock, 1, Whistler instability, J. Geophys. Res., 98, 13 325-13 333, 1993.

Zhang, Y., Matsumoto, H., and Kojima, H.: Bursts of whistler mode waves in the upstream of the bow shock: Geotail observations, J. Geophys. Res., 103, 20 529-20 540, 1998a.

Zhang, Y., Matsumoto, H., and Kojima, H.: Lion roars in the magnetosheath: The Geotail observations, J. Geophys. Res., 103, 4615-4626, 1998b. 